\title{
A neural network model for short term river flow prediction
}

\author{
R. Teschl and W. L. Randeu \\ Graz University of Technology, Department of Broadband Communications, Graz, Austria \\ Received: 3 November 2005 - Revised: 19 April 2006 - Accepted: 26 June 2006 - Published: 14 July 2006
}

\begin{abstract}
This paper presents a model using rain gauge and weather radar data to predict the runoff of a small alpine catchment in Austria. The gapless spatial coverage of the radar is important to detect small convective shower cells, but managing such a huge amount of data is a demanding task for an artificial neural network. The method described here uses statistical analysis to reduce the amount of data and find an appropriate input vector. Based on this analysis, radar measurements (pixels) representing areas requiring approximately the same time to dewater are grouped.
\end{abstract}

\section{Introduction}

In the field of weather forecasting the radar is a key instrument. The combination of radars with satellite data, automated meteorological measurements from aircraft, and with a network of ground-based meteorological instruments has been shown to provide enhanced nowcasting and short-term forecasting capabilities (Smith et al., 2002). Weather radars are mainly used in the field of short term precipitation forecasting (nowcasting). But the meteorological service is not the only field of application. Hydrological applications are gaining importance in the domain of radar technology. Due to their good spatial and temporal resolution, and their gapless spatial coverage, precipitation data acquired by weather radars offer an enormous potential for hydrological applications as well.

The following model is a further development of a rainfallrunoff model based on radar estimates of rainfall (Teschl and Randeu, 2004), applied to the Sulm-basin in the Austrian province of Styria. The previous work demonstrated the feasibility of interrelating runoff measurements of a river and radar precipitation data of the underlying catchment on

Correspondence to: $\mathrm{R}$. Teschl

(reinhard.teschl@tugraz.at) a neural network basis. The analysis showed that the radar rainfall data provided a better indication for areal precipitation and in succession for the runoff volume than a single raingauge was able to. The model presented here combines rain gauge, radar and runoff data.

\subsection{Study area}

The study area is the Sulm catchment in the south-west of Styria, Austria. The whole basin includes an area of 1105.7 square kilometres. Elevations reach from $263 \mathrm{~m}$ (above mean sea level, m.s.l.) at the watershed outlet (Leibnitz) to $2125 \mathrm{~m}$ (m.s.l.) on the Koralpe mountain range. The average watershed slope is $11.9 \%$.

Scope of this analysis is the sub-catchment Wernersdorf. This small catchment (about $35 \mathrm{~km}^{2}$ in area) is of particular interest, because as there are no more flow meters upstream, the possibilities for high water warnings for this place are limited. On the other hand the discharge measurements at Wernersdorf can be helpful to identify severe situations that may lead to hazards downstream. Our data shows that whenever the flow meter at Wernersdorf had high peaks also the flow meters downstream had maxima after a significant time lag. Figure 1 presents a map of the Wernersdorf catchment, showing the radar grid and the location of rain gauge and flow meter.

In summer this region is often affected by rain showers. Short convective storms are the dominant flood producing processes in this area (Blöschl et al., 2001). Sometimes the spatial extension of these showers is so small that a detection is only possible by weather radar, while none of the rain gauges in that area reports precipitation.

The catchment response of this part of Austria can be considered as flashy. The annual maximum daily precipitation occurs in late summer (Blöschl et al., 2001). This is the period where the maximum annual flood peaks are measured.

Published by Copernicus GmbH on behalf of the European Geosciences Union. 


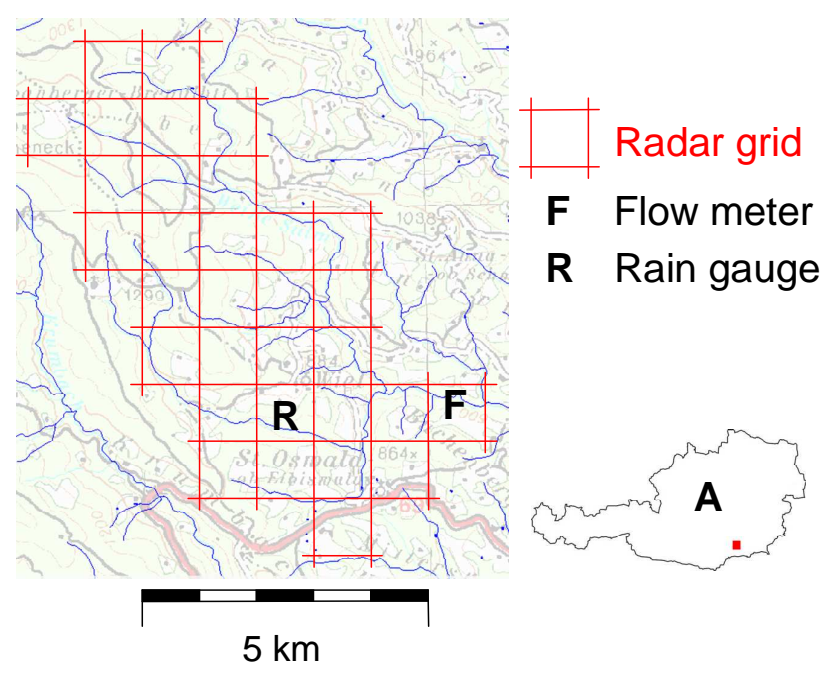

Fig. 1. Map of the Wernersdorf catchment.

\subsection{Available data}

For the processing of the neural network model rain-gauge, flow meter and radar data were available. The datasets extend over a 1-year period from January to December 2000. The temporal resolution of all datasets was assimilated to the temporal resolution of the runoff data which is $15 \mathrm{~min}$.

\subsubsection{Rain gauge and flow meter data}

Because of the specific geographic and climatic situation the rain gauge and flow meter density is quite high compared to other parts of Austria. The outflow $\left[\mathrm{m}^{3} / \mathrm{s}\right]$ is known for all tributaries in the Sulm basin at 13 different sites. The time interval between the outflow-measurements is $15 \mathrm{~min}$. Precipitation data are available from a network of rain gauges. The rain gauges are working on the tipping bucket principle with a resolution of $0.1 \mathrm{~mm}$. The temporal resolution is $15 \mathrm{~min}$. Data from 10 rain gauges are available. One rain gauge is located within the focused Wernersdorf sub-catchment. Both, rain gauge and flow meter data are officially controlled and verified by the Hydrographische Landesabteilung Steiermark (Department for Hydrography of the Province of Styria).

\subsubsection{Radar data}

To improve the spatial coverage, weather radar data from the Doppler weather radar station on Mt. Zirbitzkogel are used. The designated radar is a high-resolution C-band weatherradar. It has the following specifications:

- Altitude of the radar-station (m.s.l.): $2372 \mathrm{~m}$

- Time interval between measurements: 5 min

- 3-dB-Beamwidth: $1^{\circ}$
- Minimum elevation angle: $0.8^{\circ}$

- Spatial resolution of the volume element: $1 \mathrm{~km}^{3}$ $\left(1 \times 1 \times 1 \mathrm{~km}^{3}\right)$

- Resolution in measured reflectivity: 14 levels of rainrate, converted from reflectivity $\mathrm{Z}$ by using a fixed relationship $\left(\mathrm{Z}=200 \cdot \mathrm{R}^{1.6}\right)$

- Instrumented range: $220 \mathrm{~km}$

- Distance from the research area: to run from $42 \mathrm{~km}$ (Koralpe mountain range) to $80 \mathrm{~km}$ (Leibnitz, watershed outlet)

\section{Neural network model}

An Artificial Neural Network (ANN) is a method inspired by the human brain and nervous system. ANNs consist of a set of processing elements (neurons) operating in parallel. As the biological exemplar, the function of the ANN is determined basically by the connections between the neurons. ANNs have been used in various scientific fields to solve problems such as pattern recognition, particle identification and classification. Furthermore ANNs are a proved and efficient method to model complex input-output relationships (Aliev, 2000). They learn the relationship directly from the data being modelled. Various fields of hydrology have been investigated with success with ANNs (Adeloye and De Munari, 2006). Particularly they have been used for rainfallrunoff modelling, river flow and flood forecasting e.g. Imrie et at. (2000); Kim and Barros (2001); Toth and Brath (2002).

One of the most common neural network model is the Multi-Layer Perceptron (MLP), and this is the type of ANN used here. A MLP is a network that consists of three types of layers: input, hidden, and output layers. Patterns are introduced to the network via the input layer. In the hidden layers (one or more) the processing is done, the result for the given input pattern is produced and transmitted to the output layer. A MLP is a feed forward neural network. It is called "feed-forward" because all of the data information flows in one direction. The neurons of one layer are connected with the neurons of the following layer, there is no feedback. Here a fully connected MLP with one hidden layer is used.

The network function of an MLP is determined largely by the number of neurons in the different layers and the weighted connections between them. The product between the input $p$ and the scalar weight $w$ is calculated. Together with the scalar bias $b$, the argument of the transfer function $f$ is formed which produces the scalar output $a$.

$a=f(w p+b)$

A number of transfer or activation functions exist. Frequently used however are non-linear sigmoid functions. Multiple layers of neurons with nonlinear transfer functions like 
the sigmoid transfer function allow the network to learn nonlinear and linear relationships between input and output (Demuth and Beale, 1998). This is important for our application because the relationship between rainfall within a catchment and runoff at its outlet is known to be highly non-linear and complex (e.g. Hsu et al., 1995).

The logistic sigmoid transfer function takes the input, which may have any value between plus and minus infinity, and map the output to the range 0 to 1 . Therefore the data must be scaled so that they always fall within the specified range. The disadvantages of sigmoid activation functions in the output layer, concerning the model's ability to generalise beyond the calibration range, are given by Imrie et al. (2000). Solomatine and Dulal (2003) suggest to use an unbounded linear function in the output layer because it is able, to a certain extent, to extrapolate beyond the range of the training data. Therefore the network used here incorporates a linear function in the output layer and logistic sigmoid activation functions in the input and hidden layers.

\section{Preprocessing}

In order to make the training process of the ANN more effective the available data has been pre-processed. The goal of this process was to configure the ANN properly, more precisely, to define an input vector and a network structure that best represent the watershed behaviour.

For this purpose the dataset has been investigated with statistical methods in order to determine the correlation between input and output data. The cross-correlation is a measure of similarity of two signals. It is a function of the relative time between the signals. The cross correlation coefficient between rainfall and runoff, which was calculated by normalising the cross-correlation of the two signals, has been used to identify the time lag (offset) where the similarity is highest.

Rain gauge as well as weather radar series were investigated and the analysis showed that the time lags with the highest cross correlation coefficients between rainfall and runoff series lie between 90 to $180 \mathrm{~min}$, depending on the position within the catchment where the rainfall was measured. Table 1 shows the time lags in detail.

The analysis revealed that the correlation coefficients of rain gauge and radar measurements vary significantly. None of the radar data series obtained the maximum value of the rain gauge $(0.2747)$. The poor correlation coefficients of the radar measurements can be explained by the fact that the radar does not detect low-level precipitation below $3 \mathrm{~km}$ (m.s.l.). High reaching convective rain cells however, the dominant source of high water and floods in this area, can be detected with good visibility by the weather radar station on Mt. Zirbitzkogel.

In order to answer the question whether the time lags of the radar pixels are though comprehensible for the catch-
Table 1. Time lag with the maximum cross correlation coefficient between rainfall and runoff series. (The position number refers to the position of the $1 \mathrm{~km} \times 1 \mathrm{~km}$ radar pixels within the catchment top down line by line.)

\begin{tabular}{|c|c|c|}
\hline Measuring site & Cross correlation coeff. & Time lag [min] \\
\hline \multicolumn{3}{|l|}{ Rain gauge } \\
\hline Site no. ow 3780 & 0.2747 & 90 \\
\hline \multicolumn{3}{|l|}{ Weather radar } \\
\hline Position no.:1 & 0.1437 & 135 \\
\hline 2 & 0.1438 & 135 \\
\hline 3 & 0.1481 & 135 \\
\hline 4 & 0.1431 & 135 \\
\hline 5 & 0.1473 & 135 \\
\hline 6 & 0.1533 & 135 \\
\hline 7 & 0.1439 & 135 \\
\hline 8 & 0.1583 & 165 \\
\hline 9 & 0.1435 & 135 \\
\hline 10 & 0.1586 & 135 \\
\hline 11 & 0.1501 & 135 \\
\hline 12 & 0.1496 & 135 \\
\hline 13 & 0.1704 & 150 \\
\hline 14 & 0.1041 & 180 \\
\hline 15 & 0.1583 & 135 \\
\hline 16 & 0.1751 & 150 \\
\hline 17 & 0.1527 & 135 \\
\hline 18 & 0.1119 & 150 \\
\hline 19 & 0.1594 & 135 \\
\hline 20 & 0.1802 & 135 \\
\hline 21 & 0.1833 & 135 \\
\hline 22 & 0.1545 & 120 \\
\hline 23 & 0.1651 & 120 \\
\hline 24 & 0.1449 & 105 \\
\hline 25 & 0.1428 & 105 \\
\hline 26 & 0.1511 & 105 \\
\hline 27 & 0.1556 & 105 \\
\hline 28 & 0.1460 & 105 \\
\hline 29 & 0.1491 & 105 \\
\hline 30 & 0.1493 & 105 \\
\hline 31 & 0.1666 & 90 \\
\hline 32 & 0.1659 & 90 \\
\hline
\end{tabular}

ment, despite the low correlation coefficients, the radar pixel directly above the rain gauge was examined and compared with the time lag of the rain gauge.

The cross correlation between gauge measured rainfall and runoff becomes a maximum at a shift of $90 \mathrm{~min}$ (see Fig. 2). The radar measurement in about $3 \mathrm{~km}$ altitude above the rain gauge site (no. 24) exhibits a shift of 105 min (see Fig. 3). The difference $-15 \mathrm{~min}$ prior to the rain gauge - is connected with the temporal resolution of the time series (it is the shortest time lag which can be identified) and can be explained by the different altitude of radar and rain gauge measurements: 


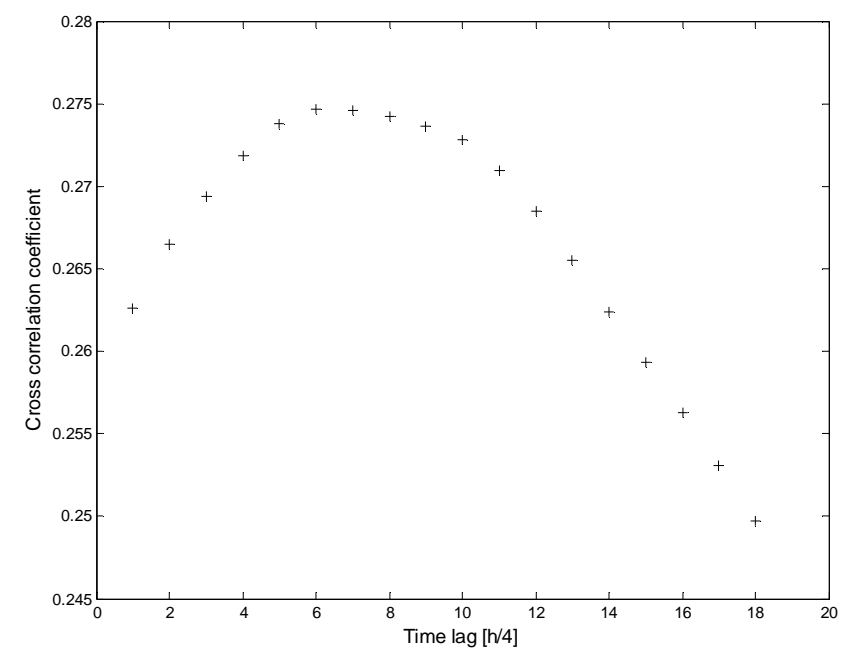

Fig. 2. Cross correlation analysis between rain-gauge and runoff measurements.

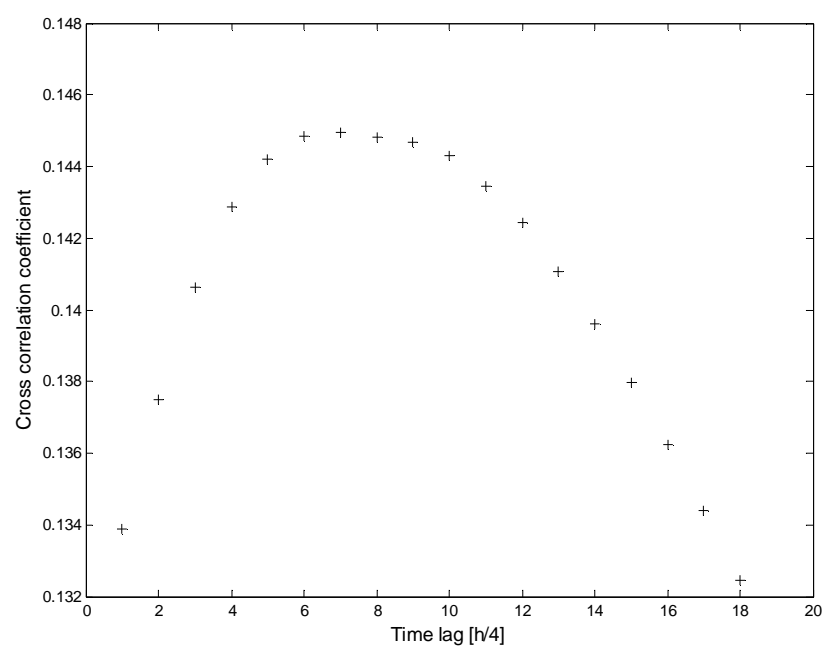

Fig. 3. Cross correlation analysis between radar (radar pixel no. 24, directly above raingauge) and runoff measurements.

aloft and on the ground. Therefore the values of the radar seem comprehensible.

The correlation analysis suggests that a forecast for 6 time lags (90 $\mathrm{min})$ is appropriate. This is the shortest shift between precipitation and runoff series that could be found.

The correlation analysis also suggests that it makes sense to define an input vector with 6 to 12 antecedent rainfall measurements depending on the time lag where the maximum correlation between runoff and rainfall was measured. But this would lead to a huge number of input parameters and an effective training would not be possible. Therefore the dimension of the input vector had to be reduced. A method to do this is for example the principal component analysis (e.g. Demuth and Beale, 1998) which eliminates those components contributing the least to the variation in the data set.
Table 2. Time lag with the maximum cross correlation coefficient between clusters and runoff series.

\begin{tabular}{rrr}
\hline Measuring site & Cross correlation coeff. & Time lag [min] \\
\hline Rain gauge & & \\
Site no. ow3780 & 0.2747 & 90 \\
Cluster no.:1 & 0.1705 & 90 \\
2 & 0.1650 & 105 \\
3 & 0.1729 & 120 \\
4 & 0.1907 & 135 \\
5 & 0.1623 & 150 \\
6 & 0.1583 & 165 \\
7 & 0.1041 & 180 \\
\hline
\end{tabular}

But this means that only a few radar measurements would be part of the input vector and the main advantage of the radar the gap less spatial coverage would be lost. The detection of small convective shower cells would not be ensured.

The method used to solve this conflict was to group radar measurements with the same time lag, leading to a smaller input vector. The radar pixels showing the same time lag to the runoff on average were summed up. This leads to groups of several pixels herein after referred to as clusters representing the amount of precipitation in this area. Table 2 shows correlation coefficient and time lag of the precipitation measurements forming the input vector. The rain gauge measurements are left unmodified. They are not grouped with radar measurements showing the same $90 \mathrm{~min}$ time lag. Cluster 1 to 7 represent summations of radar time series showing the same time lag with respect to the runoff data. The correlation coefficients of the clusters are often higher than those of the radar pixels forming the cluster. The advantage of this technique is that information of each pixel above the catchment is still represented in the dataset. Because of the bigger clusters the information where exact a small convective shower cell occurred is lost but the rainfall amount within the area represented by the cluster is available and the time lag when the rainfall shows the highest correlation with the runoff series is known.

Besides rainfall measurements, it may also be useful to present antecedent runoff measurements to the ANN. Sudheer et al. (2002) propose the partial autocorrelation to decide how much former runoff values should be included into the input vector, see Fig. 4. The time lag before the correlation falls in the $95 \%$ confidence band is used as an indicator. According to this algorithm the input vector should contain runoff values from up to 8 antecedent intervals. In our case, where a forecast for $90 \mathrm{~min}$ is made, 5 antecedent runoff measurements are not available. Therefore networks with up to 3 antecedent runoff measurements were tested. 


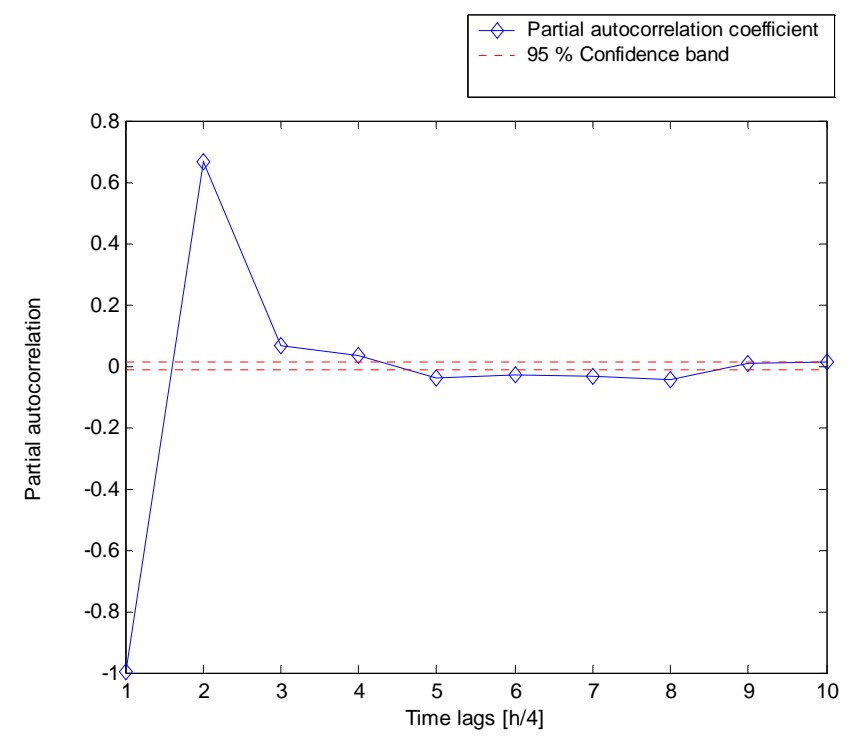

Fig. 4. Partial auto correlation analysis of the runoff data.

\section{ANN architecture}

For identifying the architecture of an ANN associated with determining the number of neurons in each layer, the trial-and-error approach is still the most common (Imrie et al., 2000; Pan and Wang, 2004; Toth et al., 2000). Some software packages perform the trial-and-error optimisation automatically. The architecture anyway is highly dependent on the problem to be solved and so no general solution can be given.

An area of conflict is that a small network may have insufficient degrees of freedom (weights and biases) to represent the relationship between rainfall and runoff, and a large network with many weights to be adapted may memorise fluctuations in the training data and is therefore not able to generalise.

Therefore the method used to determine the architecture of the ANN was to start with a small network (one hidden layer and three hidden nodes), to increase the number of nodes and to choose the network with the best performance. During the training process the error on the validation set (mean square error) was monitored. When the validation error increased the training was stopped and the minimum of the validation error was taken as indicator for the performance. Thus a network with twelve nodes in one hidden layer was determined.

Essential for a good performance of an MLP is cautious selection of the training validation and test data sets. In the present case where data of a period of one year are available the selection of the subsets for the training validation and test process is even more eminent. Eventually a method was used that ensures that each of the tree subsets contains random data from all seasons. Therefore the whole data set was

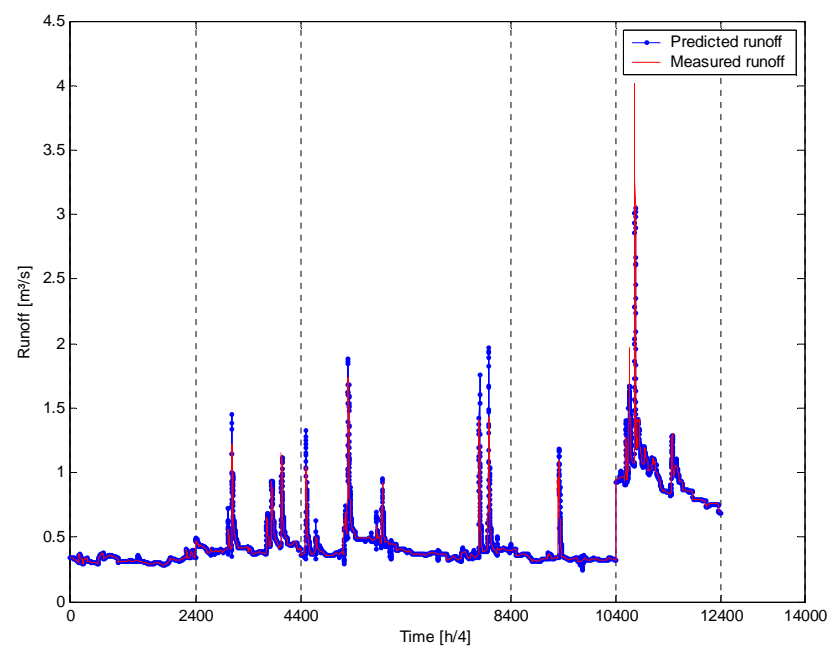

Fig. 5. Comparison between predicted and measured runoff series of the test dataset.

Table 3. RMSE and $R^{2}$ of the training set and the five subsets separately. The number of the subset refers to the occurrence in Fig. 5.

\begin{tabular}{lll}
\hline & RMSE & $\mathrm{R}^{2}$ \\
\hline Subset & & \\
1 & 0.0084 & 0.8863 \\
2 & 0.0396 & 0.8685 \\
3 & 0.0554 & 0.8566 \\
4 & 0.0365 & 0.7419 \\
5 & 0.1136 & 0.8306 \\
Overall & 0.0596 & 0.9489 \\
\hline
\end{tabular}

divided into rainfall events and their corresponding runoff hydrographs. These events where classified into the seasons they belong to and training validation and test subset where formed by randomly assigning events from all seasons to all subsets.

\section{Results and discussion}

The simulation performance of the ANN model was evaluated on the basis of Root Mean Square Error (RMSE) and $R^{2}$ efficiency coefficient by Nash and Sutcliffe (1970).

In Fig. 5 the comparison between predicted and measured runoff series can be seen. The output of the model, simulated with test data, shows a good agreement with the target concerning prediction of the time of maximum concentration. As mentioned above training validation and test data contain subsets from all seasons. In Fig. 5 the vertical grid lines separate the different subsets. 


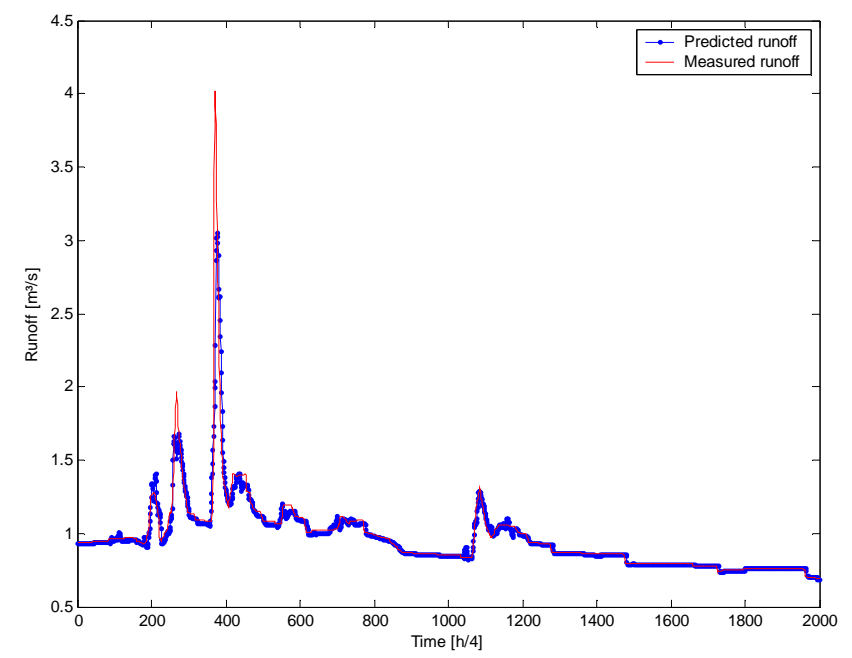

Fig. 6. Comparison between predicted and measured runoff series of the subset 5 of the testset.

Table 4. RMSE and $R^{2}$ of the validation set and the four subsets separately. The number of the subset refers to the occurrence in Fig. 7.

\begin{tabular}{lll}
\hline & RMSE & $\mathrm{R}^{2}$ \\
\hline Subset & & \\
1 & 0.0083 & 0.9497 \\
2 & 0.0919 & 0.7185 \\
3 & 0.0322 & 0.892 \\
4 & 0.1071 & 0.8694 \\
Overall & 0.0645 & 0.9412 \\
\hline
\end{tabular}

The parameters RMSE: 0.0596 and $R^{2}: 0.9489$ suggest a very good performance. In general, a $R^{2}$ value greater than 0.9 indicates a very satisfactory model performance, while a $R^{2}$ value in the range $0.8-0.9$ signifies a good performance and values less than 0.8 indicate an unsatisfactory model performance (Coulibaly and Baldwin, 2005).

The $R^{2}$ value has to be treated with caution, because it contains the mean of the observed runoff values. Because of high runoff values of the last subset of the training dataset the mean value over the whole training set is 0.4884 . Table 3 gives the RMSE and $R^{2}$ values for the five subsets of the training data.

Table 3 shows that the performance of all subsets except subset 4 can be considered as good. The high RMSE of subset 5 is due to the underestimation of the highest peak. Figure 6 shows this subset in detail.

Figure 6 shows that the dynamics in the hydrograph are captured quite well by the model, while the highest peak is underestimated. The underestimation is believed to result from the fact that the training dataset did not contain such

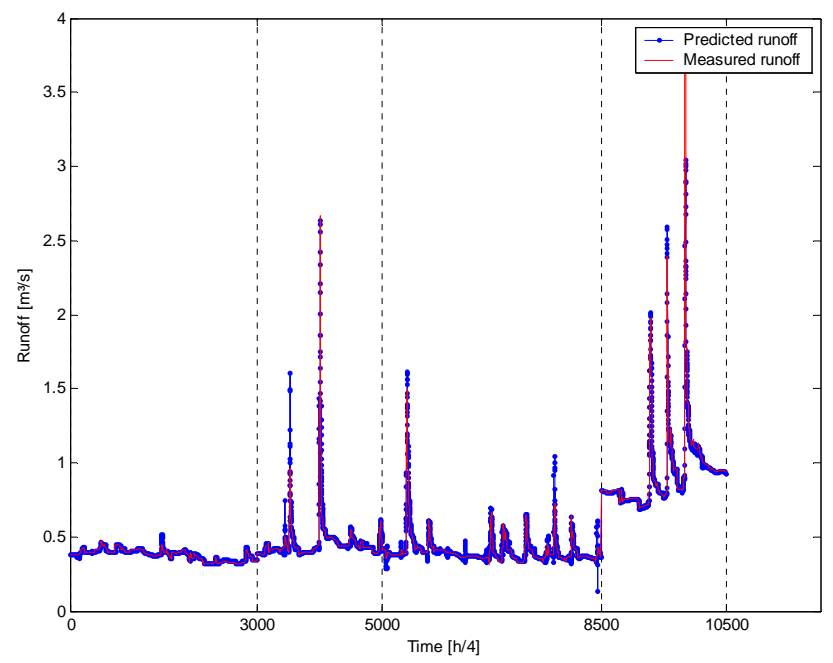

Fig. 7. Comparison between predicted and measured runoff series of the validation dataset.

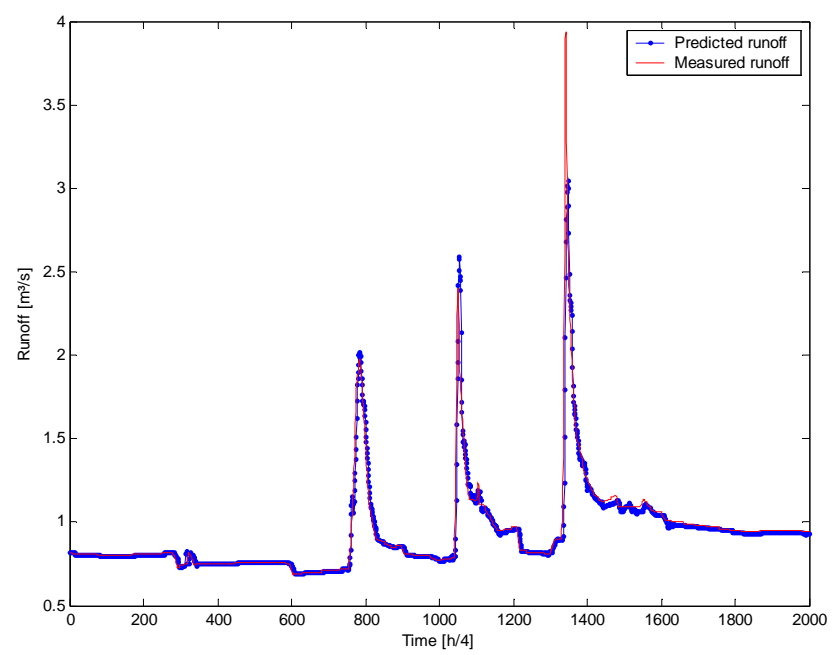

Fig. 8. Comparison between predicted and measured runoff series of the subset 4 of the validation set.

high discharge values. This assumption is supported by an analysis of the vaildation dataset.

Again the highest peak is underestimated. Obviously the effect the unbounded linear function in the output layer has, to help the ANN extrapolate beyond the range of the training data, is not significant. Figure 8 shows the affected subset 4 in detail.

Concerning RMSE and $R^{2}$ the performance of the test and validation set is more or less equal. The validation subset shown in Fig. 8 also has with 0.1071 a high RMSE value. Table 4 shows the details.

Acknowledgements. The authors would like to thank W. Verwüster form the Hydrographische Landesabteilung for providing the flow meter and rain gauge data and the anonymous reviewers for 
their helpful suggestions and valuable comments on the original manuscript.

Edited by: P. P. Alberoni

Reviewed by: two referees

\section{References}

Adeloye, A. J. and De Munari, A.: Artificial neural network based generalized storage-yield-reliability models using the Levenberg-Marquardt algorithm, J. Hydrol., 326(1-4), 215-230, 2006.

Aliev, R., Bonfig, K. W., and Aliew, F.: Soft Computing: Eine grundlegende Einführung, Verlag Technik, Berlin, 2000.

Blöschl, G., Merz, R., and Piock-Ellena, U.: Flash-Flood Risk Assessment under the Impacts of Land Use Changes and River Engineering Works, Final Report, Institute of Hydraulics, Hydrol. Water Resour. Manage., Vienna University of Technology, 2001.

Coulibaly, P. and Baldwin, C. K.: Nonstationary hydrological time series forecasting using nonlinear dynamic methods, J. Hydrol., 307, 164-174, 2005.

Demuth, H. and Beale, M.: MATLAB Neural Networks Toolbox User's Guide, Fifth Printing - Version 3, 1998.

Hsu, K., Vijai Gupta, H., and Sorooshian, S.: Artificial neural network modeling of the rainfall-runoff process, Water Resour. Res., 31(10), 2517-2530, 1995.

Imrie, C. E., Durucan, S., and Korre, A.: River flow prediction using artificial neural networks: generalisation beyond the calibration range, J. Hydrol., 233, 138-153, 2000.
Kim, G. and Barros, A. P.: Quantitative flood forecasting using multisensor data and neural networks, J. Hydrol., 246, 45-62, 2001.

Nash, J. E. and Sutcliffe, J. V.: River flow forecasting through conceptual models I: A discussion of principles, J. Hydrol., 10, 282290, 1970.

Pan, T.-Y. and Wang, R.-Y.: State space neural networks for short term rainfall-runoff forecasting, J. Hydrol., 297, 34-50, 2004.

Solomatine, D. P. and Dulal, K. N.: Model trees as an alternative to neural networks in rainfall-runoff modelling, Hydrological Sciences - Journal des Sciences Hydrologiques, 48, 399-411, 2003.

Sudheer, K. P., Gosain, A. K., and Ramasastri, K. S.: A data driven algorithm for constructing ANN based rainfall-runoff models, Hydrol. Processes, 16, 1325-1330, 2002.

Smith, P. L. (Chairman), Atlas, D., Bluestein, H. B., Chandrasekar, N. V., et al.: Weather Radar Technology Beyond NEXRAD, Committee on Weather Radar Technology Beyond NEXRAD, National Research Council, ISBN: 0-309-08466-0, 96 pages, 2002.

Teschl, R. and Randeu, W. L.: An Artificial Neural Networkbased Rainfall-Runoff Model using gridded Radar Data. Third European Conference on Radar in Meteorology and Hydrology (ERAD), Visby, Island of Gotland, Sweden, Paper nr.: ERAD3A-00163, 2004.

Toth, E., Brath, A., and Montanari, A.: Comparison of short-term rainfall prediction models for real-time flood forecasting, J. Hydrol., 239, 132-147, 2000. 